\title{
Exhaled Breath Condensate pH as a Non-invasive Measure of Inflammation in Non-CF Bronchiectasis
}

\author{
A. Shoemark and R. Wilson \\ Host Defence Unit, Royal Brompton Hospital, Sydney Street, London SW3 6NP, UK \\ Correspondence should be addressed to R. Wilson, r.wilson@rbht.nhs.uk
}

Received 4 August 2011; Accepted 28 August 2011

Academic Editors: A. Sharafkhaneh and M. Tatar

Copyright (c) 2011 A. Shoemark and R. Wilson. This is an open access article distributed under the Creative Commons Attribution License, which permits unrestricted use, distribution, and reproduction in any medium, provided the original work is properly cited.

\begin{abstract}
Bronchiectasis is characterised by neutrophilic bronchial inflammation. Direct measurement of lung inflammation would be useful to assess disease activity, guide need for treatment, and monitor response. The aim of this study was to test whether exhaled breath condensate $(\mathrm{EBC}) \mathrm{pH}$, a simple noninvasive test, provides a clinically useful measure of inflammation in the lungs of patients with bronchiectasis. 96 consecutively referred patients were studied when clinically stable, 20 followed up over two years, and a further 22 patients seen during an exacerbation. Subjects breathed tidally for 10 minutes into a condensing chamber (Ecoscreen, Erich Jaeger, Hoechberg, Germany). pH in EBC was measured immediately using a pH probe. In a representative group of 25 patients samples were deaerated with argon gas. This was to control for variations in $\mathrm{pH}$ ex vivo by removing $\mathrm{CO}_{2}$. EBC was acidic in bronchiectasis patients $(6.79 \pm 0.72)$ compared to controls $(7.08 \pm 0.69)$ and primary ciliary dyskinesia patients $(7.24 \pm 0.53)$. pH was related to lung volume but not disease severity. Repeated measures show EBC pH changes with symptoms. EBC is further acidified during an exacerbation of bronchiectasis $(6.44 \pm 0.72)$, this acidification persists following treatment $(6.09 \pm 0.80)$. EBC $\mathrm{pH}$ is not sufficiently sensitive or specific to monitor patients' health status or provide information to inform acute treatment decisions.
\end{abstract}

\section{Introduction}

Patients with bronchiectasis have airway dilatation due to a structural abnormality of the bronchial wall that leads to delayed mucociliary clearance and predisposes the lung to bacterial infection [1]. In severe disease, infection is often persistent leading to chronic inflammation causing further damage to the lung tissue [2]. The levels of inflammation in stable state bronchiectasis correlate with a reduction in patient's quality of life [3]. Monitoring inflammation is an important part of management of bronchiectasis patients in order to prevent disease progression and enhance quality of life. Current markers of inflammation such as blood, sputum markers, and bronchoscopy can be indirect, variable and invasive, respectively.

Exhaled breath condensate (EBC) is the collection of breath as a liquid by exhaling through a cooled tube. EBC contains microdroplets of extracellular lining fluid, an important first line of the lung's defence system. EBC is a simple non-invasive test to perform making it an attractive technique for potentially monitoring oxidative stress and lung inflammation.

A range of markers can be measured in EBC. $\mathrm{pH}$ and ammonia are simple markers which are easy to measure. Both are important within the respiratory system as mediators of host defence and have potential to reflect neutrophilic inflammation in the lungs of patients with bronchiectasis. $\mathrm{pH}$ shows good reproducibility [4] and has previously been shown to be reduced in 20 patients with bronchiectasis compared with normal controls and mild asthmatics [5]. The reduced $\mathrm{pH}$ values correlated with airway neutrophilia, EBC hydrogen peroxide, and $\mathrm{FEV}_{1}$. $\mathrm{pH}$ was reduced further in patients with Pseudomonas aeruginosa colonisation suggesting $\mathrm{pH}$ may be a potential inflammatory marker in bronchiectasis. Ammonia is a potential regulator of $\mathrm{pH}[6]$ and has not been studied previously in $\mathrm{EBC}$ of non-CF bronchiectasis patients. We aim to establish the value of EBC $\mathrm{pH}$ and its relationship to ammonia as an inflammatory marker in bronchiectasis whilst patients are stable and during an exacerbation of the condition. 
1.1. Aim. This paper aims to test the hypothesis that $\mathrm{EBC} \mathrm{pH}$ will provide clinically useful direct measures of inflammation in the lungs of patients with bronchiectasis.

To address this hypothesis, we assess EBC in a large group of patients with bronchiectasis compared to healthy controls. $\mathrm{EBC} \mathrm{pH}$ is compared with existing measures of inflammation, $\mathrm{EBC}$ ammonia as a potential $\mathrm{pH}$ regulator, disease activity and severity, and longitudinal assessments are made following patients up every 6 months for two years. EBC is also measured during and after an acute exacerbation of bronchiectasis requiring IV antibiotics.

\section{Methods}

2.1. Ethics. This study was approved by the Royal Brompton Hospital ethics committee and subjects gave written informed consent to take part.

\subsection{Study Design}

2.2.1. Consecutive Referral Cross-Sectional Study. Consecutive patients were studied whilst they attended the hospital for a program of tests designed to investigate the cause and severity of their bronchiectasis. This protocol has been previously described [7]. Patients were asked to perform EBC collection. $\mathrm{pH}$ and ammonia were measured and the results compared to those of twenty healthy controls.

Results were compared with age, gender, height, weight, patient's sputum (including, sputum volume, purulence, eosinophil counts, and bacteriology), peripheral blood inflammatory markers (white cell count, neutrophils, ESR and CRP), quality of life (as judged by SGRQ score), exercise capacity (measure by shuttle walking test), exhaled nitric oxide $\left(\mathrm{FE}_{\mathrm{NO}}\right)$, lung function $\left(\mathrm{FEV}_{1}\right.$ and $\%$ predicted), and disease severity on CT scan.

2.2.2. Exacerbation Study. EBC was collected within 24 hours of admission to hospital, for an infective exacerbation of bronchiectasis requiring intravenous antibiotics, and within 24 hours of discharge. Antibiotics were chosen by a physician who was guided by current or previous sputum sensitivities. The time of discharge was dependent on clinical response to treatment.

2.2.3. Two Year Longitudinal Study. Patients were recruited from the outpatient department when stable. They were seen every 6 months and each time they visited clinic for the following two years.

At each visit the patient completed $\mathrm{EBC}$ collection, $\mathrm{FE}_{\mathrm{NO}}$ measurement, $\mathrm{FEV}_{1}$, and a SGRQ. Other tests such as blood markers of inflammation and sputum bacteriology were performed when clinically indicated. Treatment was also given when clinically indicated.

2.3. Study Subjects. Patients with bronchiectasis were recruited from the infection firm at the Royal Brompton hospital. Bronchiectasis was confirmed by high-resolution CT scan according to standard criteria [8].
Hospital staff of similar age and gender to the bronchiectasis group were recruited as normal controls. Study group demographics are shown in Table 1.

\subsection{Experimental Tests}

2.4.1. EBC Collection. Subjects were asked to sit breathing tidally for 10 minutes through a non-rebreathing valve with saliva trap into a condensing chamber. (Ecoscreen, Erich Jaeger, Hoechberg, Germany). The exhaled breath was cooled in a metal chamber so that it condensed. It was then collected as a liquid in an inert disposable collection vessel at the bottom of the metal chamber. Patients were instructed to remove their mouth away from the mouthpiece if they needed to cough, yawn, or sneeze, if subjects needed to swallow saliva or if their breathing became uncomfortable. Nose clips were not used.

2.4.2. $p H$ Measurement. The condensate was stirred manually and $\mathrm{pH}$ was measured immediately using a small combination $\mathrm{pH}$ probe (BDH, Poole, $\mathrm{UK}$ ) and bench $\mathrm{pH}$ meter (Hanna Instruments, UK). The bench meter was calibrated before each measurement with 4.01, 7.01, and 10.01 standards and adjusted according to the temperature of the sample, measured with a small hand-held thermometer. Reproducibility of this measurement was checked once a day over three days in a group of 10 patients. All patients were able to perform the EBC measurement every day for three days. EBC $\mathrm{pH}$ reliability coefficient was 0.66 and nonsignificant variation was calculated to be \pm 0.56 . In a representative group of 25 patients, samples were deaerated with argon gas. This was to control for variations in $\mathrm{pH}$ ex vivo by removing $\mathrm{CO}_{2}$. This results in a $\mathrm{pH}$ which is stable for a long time frame [9]. Argon gas (BOC speciality gases, UK) was bubbled through the sample at a rate of $350 \mathrm{~mL} / \mathrm{min}$ until the $\mathrm{pH}$ of the sample stabilised for more than 30 seconds. This was achieved using a series of regulators and a flow controller linked to a small inert plastic tube which was inserted directly into the condensate.

2.4.3. Ammonia Measurement. Ammonia content was measured using an $\mathrm{NH} 4+$ electrode in conjunction with a double junction reference electrode (Thermo Orion, USA) connected to a bench $\mathrm{mV}$ meter (Hanna Instruments, UK). An analate addition method was used as described by Gaston et al. (2002) [10].

2.5. Data Analysis. The data was evaluated using commercially available statistical analysis software (SPSS). Differences were considered to be significant when probability values were less than or equal to 0.05 . Where data was normally distributed, values are reported as mean and standard deviation. Where data distributions differed from normal values are reported as median and variation is reported by interquartile range. The natural log of the value was used to normalise the data distribution for statistical analysis. A student's $t$-test or ANOVA was used to compare groups and linear regression analysis used to assess relationship between variables. 
TABLE 1: Study group demographics.

\begin{tabular}{lcccc}
\hline & $\begin{array}{c}\text { Consecutive referrals with } \\
\text { bronchiectasis }\end{array}$ & $\begin{array}{c}\text { Two-year longitudinal } \\
\text { assessment }\end{array}$ & $\begin{array}{c}\text { Exacerbation of } \\
\text { bronchiectasis }\end{array}$ & Control subjects \\
\hline$n$ & 63 & 20 & 20 & 30 \\
Age in years median (IQR) & $50(15)$ & $52(8)$ & $54(7.5)$ & $42(24)$ \\
Male (\% group) & $19(30)$ & $10(50)$ & $10(32)$ & $8(27)$ \\
Smoking status & $54 / 9 / 0$ & $20 / 0 / 0$ & $19 / 1 / 0$ & $30 / 0 / 0$ \\
(non/ex/current) & $70.9(19.7)$ & $60.7(26.0)$ & N/A & N/A \\
FEV F $_{1} \%$ pred & $50.9(25.9)$ & $41.9(13.0)$ & N/A & N/A \\
SGRQ total & & & & \\
\hline
\end{tabular}

SGRQ: St George’s Respiratory Questionnaire.

\section{Results}

3.1. EBC in Consecutive Referrals Compared to Healthy Controls. Ninety six consecutive referrals had EBC collected and $\mathrm{pH}$ and ammonia measurements taken. Their results were compared to those of 20 healthy controls of similar age and gender. Patients with non-CF, non-PCD bronchiectasis have acidified breath condensate. These results are shown in Figure 1. Patients with bronchiectasis caused by primary ciliary dyskinesia (PCD) had $\mathrm{pH}$ similar to normal controls. This could be related to low exhaled nitric oxide in this group 6.1 (4.5) ppb versus 13.9 (7.0) in controls $(P<0.05) .22$ patients did not have evidence of bronchiectasis on CT scan. Their EBC pH was not significantly different to those patients with bronchiectasis.

There was no significant difference in ammonia levels between bronchiectasis patients $0.13 \mathrm{mM}(0.20)$ and normal controls $0.21 \mathrm{mM}(0.31), P=0.31$. However, there was a weak correlation between EBC ammonia and $\mathrm{pH} r=0.42$, $P=0.02$.

Twelve subjects did not produce a condensate sample. In three of these subjects the measurement was repeated on a separate day, and still no condensate was collected. This may be due to nose breathing despite instruction to breath through the mouth.

EBC measurements of $\mathrm{pH}$ and ammonia were compared with a number of demographic factors and measures of disease activity and severity, as described in Section 2.2.1. There was no relationship between any of the factors measured and EBC $\mathrm{pH}$ or ammonia levels.

These studies were repeated in a group of 25 patients in which EBC was deaerated with argon gas. This was to control for variations in $\mathrm{pH}$ ex vivo by removing $\mathrm{CO}_{2}$. This results in a more stable $\mathrm{pH}$ result [9]. When EBC was deaerated, a correlation between EBC $\mathrm{pH}$ and height and consequently a weak relationship with $\mathrm{FEV}_{1}(r=0.43, P=0.05)$ and a difference in $\mathrm{pH}$ between genders were revealed. The group consisted of eleven males and fourteen females. Females had significantly more acidic pH mean (SD) 7.20 (0.23) than the males $7.49(0.21), P=0.02$ (shown in Figure 2). In the control group in which EBC was deaerated $(n=20)$, there was no difference in $\mathrm{pH}$ between males and females and no relationship with height $(r=0.08, P=0.78)$. Male reported $n=7$ mean (SD) $7.43(0.18)$, and female $n=13$ mean (SD) 7.63 (0.16), $P=0.11$.

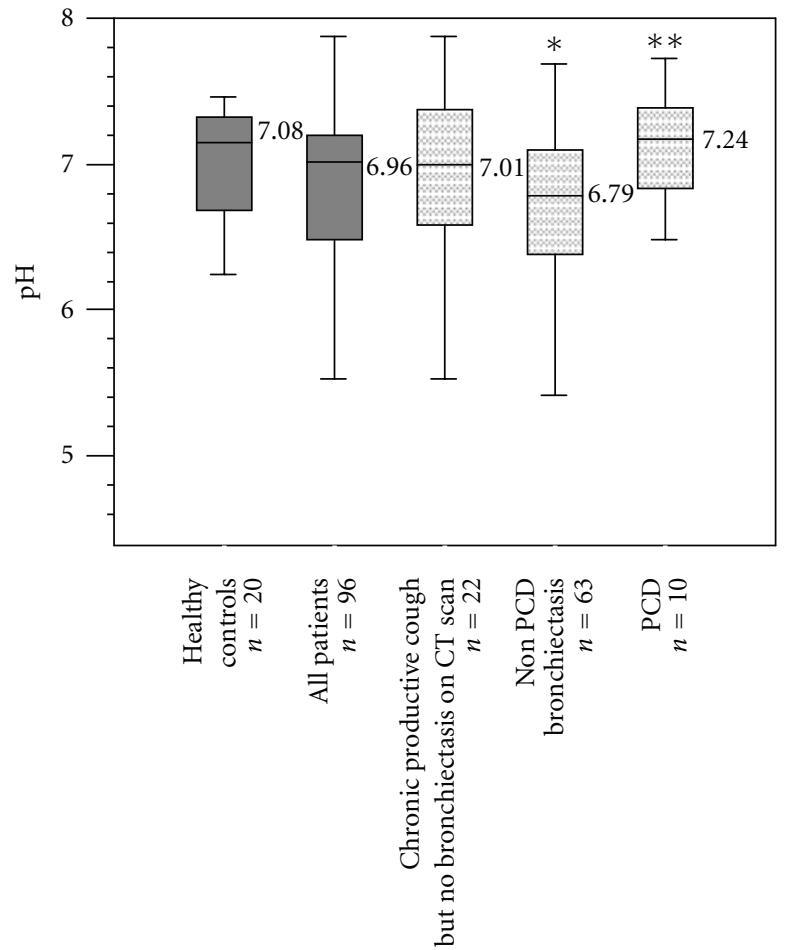

FIGURE 1: $\mathrm{pH}$ of EBC in patients with bronchiectasis compared to primary ciliary dyskinesia, chronic cough, and normal controls. ${ }^{*} P=0.04$ when comparing non-PCD bronchiectasis patients with healthy controls. ${ }^{* *} P=0.01$ when comparing PCD patients with non-PCD bronchiectasis patients. 1 patient with CF was not included in the graphical representation of the breakdown of all patients.

3.2. Exacerbation of Bronchiectasis. EBC $\mathrm{pH}$ was acidic in 20 patients with bronchiectasis on admission to hospital for an exacerbation of their condition. The median (IQR) $\mathrm{pH}$ was 6.44 (0.72). pH did not normalise following treatment (as shown in Figure 3). Predictive value of $\mathrm{pH}$ for an exacerbation was calculated as $19 \%$ sensitive and $92 \%$ specific at $\mathrm{pH}<$ 5.85. There was no relationship between EBC $\mathrm{pH}$, ammonia, or $\mathrm{FE}_{\mathrm{NO}}$ at the time patients were admitted to hospital. At the time of discharge there was a negative correlation between $\mathrm{FE}_{\mathrm{NO}}$ and $\mathrm{EBC} \mathrm{pH} r=-0.48, P=0.02$. Blood inflammatory markers were elevated at admission and significantly reduced at discharge. There was no change in EBC ammonia (data not shown). 


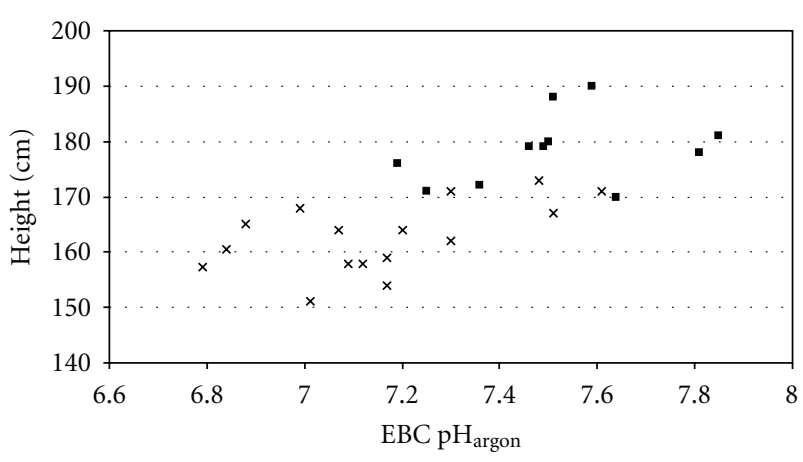

FIGURE 2: Increased $\mathrm{pH}$ in EBC deaerated with argon gas from patients with bronchiectasis according to their height and gender. $\times=$ female $\boldsymbol{\square}=$ male. $r=0.68, P=0.003$.

3.3. Two-Year Longitudinal Assessment. EBC pH was measured in 20 patients over a course of 2 years. EBC $\mathrm{pH}$ changed in relation to SGRQ total score over the course of the study: $\beta$ coefficient $=-0.007, P=0.017$. This was due to a relationship between $\mathrm{pH}$ and the symptom component $\beta=-0.007$, $P=0.014$ of the questionnaire. There was no relationship with lung function.

\section{Discussion}

A large group of patients with well-defined bronchiectasis in terms of aetiology, bacteriology, and disease severity was established and EBC pH and ammonia were successfully measured. EBC can easily be collected in patients with symptoms of bronchiectasis regardless of disease activity or severity. $\mathrm{EBC} \mathrm{pH}$ is more acidic in bronchiectasis than in healthy controls of similar age and gender. However, acidification was not related to structural damage, as patients with bronchial suppuration but no structural damage had similar EBC $\mathrm{pH}$ and there was no difference in $\mathrm{EBC} \mathrm{pH}$ in patients with more severe bronchiectasis, judged by CT and lung function. This acidification is important since many processes in the respiratory system are $\mathrm{pH}$ dependant. These include bronchoconstriction and several host defence mechanisms which may be of importance when considering the bronchiectatic lung. For example, low $\mathrm{pH}$ results in impairment of ciliary motility, increase of airway mucus viscosity, and damage of the airway epithelium [11-15].

EBC $\mathrm{pH}$ was similar in patients with primary ciliary dyskinesia to normal controls and was less acidic when compared with other causes of bronchiectasis. This may represent less inflammation in the primary ciliary dyskinesia disease group compared to other patients. Alternatively, it may be related to low $\mathrm{FE}_{\mathrm{NO}}$ in this condition and thus explain one of the sources of low $\mathrm{pH}$ in the other patients where $\mathrm{FE}_{\mathrm{NO}}$ is raised [16]. Available nitrite can be converted to nitrous acid which lowers $\mathrm{pH}$. Where there is no available nitric oxide as in PCD then nitrous acid may not be formed and the expirate may be less acidic. Indeed there was a negative correlation between $\mathrm{FE}_{\mathrm{NO}}$ and $\mathrm{EBC} \mathrm{pH}$ at discharge after treatment for an infective exacerbation of bronchiectasis. This relationship was not present in stable patients or at the start of an exacerbation. This suggests both $\mathrm{EBC} \mathrm{pH}$ and $\mathrm{FE}_{\mathrm{NO}}$ may be related

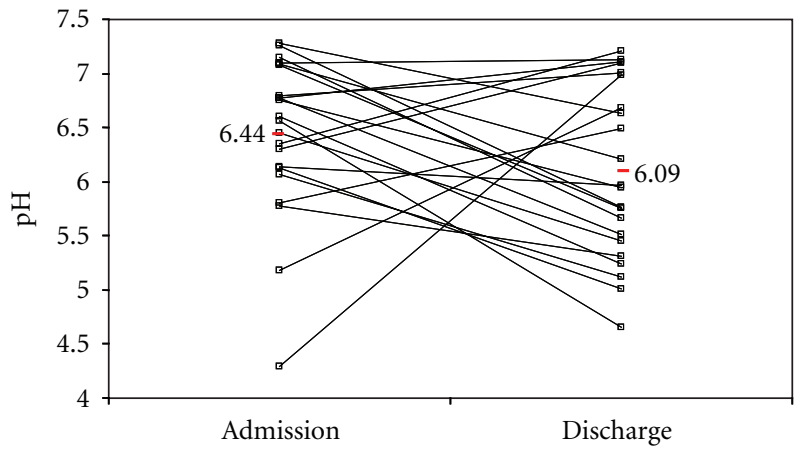

FIGURE 3: No change in $\mathrm{pH}$ of EBC in patients within 24 hours of admission to hospital for an exacerbation of bronchiectasis and at discharge from hospital following a course of intravenous antibiotics.

once the exacerbation and other potential contributing factors have been brought under control by treatment.

Some non-inflammatory patient factors influenced the EBC measurement. There is a relationship between EBC pH and patient height which may relate to lung size. EBC $\mathrm{pH}$ was more acidic in patients with reduced $\mathrm{FEV}_{1}$ although there was no relationship with $\mathrm{FEV}_{1} \%$ predicted. Also there is a difference between $\mathrm{pH}$ in males and females, females having more acidic EBC pH. These should be considered and corrected for if the measurement is to be used as a clinical tool. Height is related closely to lung size and volume and therefore there may be a larger surface area over which acids can be buffered.

Patients were asked to breath into the condenser for 10 minutes. The volume of condensate differed from $0 \mathrm{~mL}$ to up to $5 \mathrm{~mL}$. Therefore it could be considered that the concentration of the compounds of interest may be altered by the amount of water vapour produced to cause dilution. This could vary according to the temperature of the condenser which was not constant, the breathing pattern, and lung volumes of the subject. A method to assess dilution of the condensate has been proposed [17]. However another group have shown $\mathrm{pH}$ to be stable regardless of dilution with airway water vapour, temperature collection, age of subject, or breathing pattern during collection [9].

Longitudinal measurements showed that within individuals the change in EBC $\mathrm{pH}$ related to symptoms (SGRQ symptom score) and $\mathrm{EBC} \mathrm{pH}$ is further acidified during an exacerbation of bronchiectasis, but not by more than the normal variation in a stable patient over 3 days $(0.56)$. The specificity and sensitivity for $\mathrm{EBC} \mathrm{pH}$ to predict an exacerbation and indicate the need for treatment were low, suggesting that EBC $\mathrm{pH}$ does not provide a clinically useful tool for measuring inflammation in bronchiectasis.

There is no difference in EBC ammonia between patients with bronchiectasis and healthy volunteers. In addition there is no correlation between EBC ammonia and any other measurements of disease activity or inflammation in bronchiectasis, and no change during an exacerbation. These findings suggest that EBC ammonia would not provide a clinically useful direct measure of inflammation in the lungs of patients with bronchiectasis. However ammonia levels correlate 
with $\mathrm{EBC} \mathrm{pH}$ and this may provide information about the mechanism for reduced $\mathrm{pH}$ in patients with bronchiectasis [5].

Significant acidification of EBC $\mathrm{pH}$ has been described in other inflammatory airway diseases, such as asthma $[6,18]$, COPD [6], and CF [4] compared to healthy control subjects. This acidification relates to oxidative stress and can normalise with corticosteroid or antibiotic treatment. There was no correlation between EBC pH and disease severity on lung function, suggesting EBC is not a sensitive marker of disease severity in bronchiectasis. Likewise in CF no relationship between $\mathrm{FEV}_{1}$ and $\mathrm{EBC} \mathrm{pH}$ has been found [4]. In contrast, a relationship in COPD and in asthma between $\mathrm{pH}$ and lung function has been identified [6]. This may be related to the area that is being assessed whilst measuring EBC. Tidal breathing into the condenser is likely to result in the majority of the aerosolised droplets originating from the proximal airways. It may be in bronchiectasis and CF that other parts of the lung are acidified with worsening lung disease but that this acidification is not measured using the EBC method described. EBC is most likely generated from the proximal airways due to the gentle tidal breathing manoeuvre with which it is collected. Therefore, $\mathrm{EBC} \mathrm{pH}$ may be related to patient symptom score as a reflection of cough. Many bronchiectasis patients following an exacerbation continue to have an irritable cough and bronchial hypereactivity. This has been thought to be due to damaged epithelium but would be aggravated by the acidic $\mathrm{pH}$ which persists after treatment of the exacerbation [19].

In summary EBC is acidified in bronchiectasis patients compared with healthy controls. EBC pH relates to lung volume in patients with bronchiectasis but is not associated with severity of disease. Repeated measures over two years show change in EBC pH correlate with change in health status, which may be related to the relationship between cough and $\mathrm{pH}$. EBC is further acidified during an exacerbation of bronchiectasis which persists following treatment. This could contribute to persistence of cough following an exacerbation. The drawbacks of EBC analysis lie in the anatomical sources of the compounds measured in EBC are not well defined and dilution of the condensate may affect results. $\mathrm{EBC} \mathrm{pH}$ is not sufficiently specific or sensitive to monitor inflammatory status or provide information to inform acute treatment decisions.

\section{Competing Interests}

The authors declare no conflict of interests.

\section{Acknowledgments}

The authors would like to thank Michael Roughton and Winston Banya from the Royal Brompton hospital statistical advice service for statistical advice.

\section{References}

[1] R. Wilson, "Bronchiectasis," in Respiratory Medicine, J. G. Gibson, D. M. Geddes, U. Costabel, P. J. Sterele, and B. Corrin,
Eds., pp. 1445-1464, Elsevier Science Ltd, Amsterdam, The Netherlands, 2003.

[2] R. Wilson, R. B. Dowling, and A. D. Jackson, "The biology of bacterial colonization and invasion of the respiratory mucosa," European Respiratory Journal, vol. 9, no. 7, pp. 1523-1530, 1996.

[3] C. B. Wilson, P. W. Jones, C. J. O’Leary, D. M. Hansell, P. J. Cole, and R. Wilson, "Effect of sputum bacteriology on the quality of life of patients with bronchiectasis," European Respiratory Journal, vol. 10, no. 8, pp. 1754-1760, 1997.

[4] S. Tate, G. MacGregor, M. Davis, J. A. Innes, and A. P. Greening, "Airways in cystic fibrosis are acidified: detection by exhaled breath condensate," Thorax, vol. 57, no. 11, pp. 926929, 2002.

[5] K. Kostikas, G. Papatheodorou, K. Ganas, K. Psathakis, P. Panagou, and S. Loukides, "pH in expired breath condensate of patients with inflammatory airway diseases," American Journal of Respiratory and Critical Care Medicine, vol. 165, no. 10, pp. 1364-1370, 2002.

[6] J. F. Hunt, E. Erwin, L. Palmer et al., "Expression and activity of $\mathrm{pH}$-regulatory glutaminase in the human airway epithelium," American Journal of Respiratory and Critical Care Medicine, vol. 165, no. 1, pp. 101-107, 2002.

[7] A. Shoemark, L. Ozerovitch, and R. Wilson, "Aetiology in adult patients with bronchiectasis," Respiratory Medicine, vol. 101, no. 6, pp. 1163-1170, 2007.

[8] D. P. Naidich, D. I. McCauley, and N. F. Khouri, "Computed tomography of bronchiectasis," Journal of Computer Assisted Tomography, vol. 6, no. 3, pp. 437-444, 1982.

[9] J. Vaughan, L. Ngamtrakulpanit, T. N. Pajewski et al., "Exhaled breath condensate $\mathrm{pH}$ is a robust and reproducible assay of airway acidity," European Respiratory Journal, vol. 22, no. 6, pp. 889-894, 2003.

[10] B. Gaston, F. Ratjen, J. W. Vaughan et al., "Nitrogen redox balance in the cystic fibrosis airway: effects of antipseudomonal therapy," American Journal of Respiratory and Critical Care Medicine, vol. 165, no. 3, pp. 387-390, 2002.

[11] C. K. Luk and M. J. Dulfano, "Effect of $\mathrm{pH}$, viscosity and ionic-strength changes on ciliary beating frequency of human bronchial explants," Clinical Science, vol. 64, no. 4, pp. 449451, 1983.

[12] B. Holma and P. O. Hegg, "pH- and protein-dependent buffer capacity and viscosity of respiratory mucus. Their interrelationships and influence on health," Science of the Total Environment, vol. 84, pp. 71-82, 1989.

[13] E. C. Veerman, M. Valentijn-Benz, and A. V. Nieuw Amerongen, "Viscosity of human salivary mucins: effect of $\mathrm{pH}$ and ionic strength and role of sialic acid," Journal de biologie buccale, vol. 17, no. 4, pp. 297-306, 1989.

[14] C. Clary-Meinesz, J. Mouroux, J. Cosson, P. Huitorel, and B. Blaive, "Influence of external $\mathrm{pH}$ on ciliary beat frequency in human bronchi and bronchioles," European Respiratory Journal, vol. 11, no. 2, pp. 330-333, 1998.

[15] F. L. M. Ricciardolo, V. Rado, L. M. Fabbri, P. J. Sterk, G. U. Di Maria, and P. Geppetti, "Bronchoconstriction induced by citric acid inhalation in guinea pigs: role of tachykinins, bradykinin, and nitric oxide," American Journal of Respiratory and Critical Care Medicine, vol. 159, no. 2, pp. 557-562, 1999.

[16] A. Shoemark and R. Wilson, "Bronchial and peripheral airway nitric oxide in primary ciliary dyskinesia and bronchiectasis," Respiratory Medicine, vol. 103, no. 5, pp. 700-706, 2009.

[17] R. M. Effros, J. Biller, B. Foss et al., "A simple method for estimating respiratory solute dilution in exhaled breath condensates," American Journal of Respiratory and Critical Care Medicine, vol. 168, no. 12, pp. 1500-1505, 2003. 
[18] J. F. Hunt, K. Fang, R. Malik et al., "Endogenous airway acidification: implications for asthma pathophysiology," American Journal of Respiratory and Critical Care Medicine, vol. 161, no. 3, pp. 694-699, 2000.

[19] R. H. Lowry, A. M. Wood, and T. W. Higenbottam, "Effects of $\mathrm{pH}$ and osmolarity on aerosol-induced cough in normal volunteers," Clinical Science, vol. 74, pp. 373-376, 1988. 


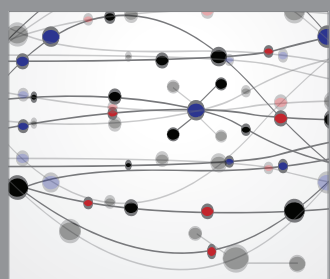

The Scientific World Journal
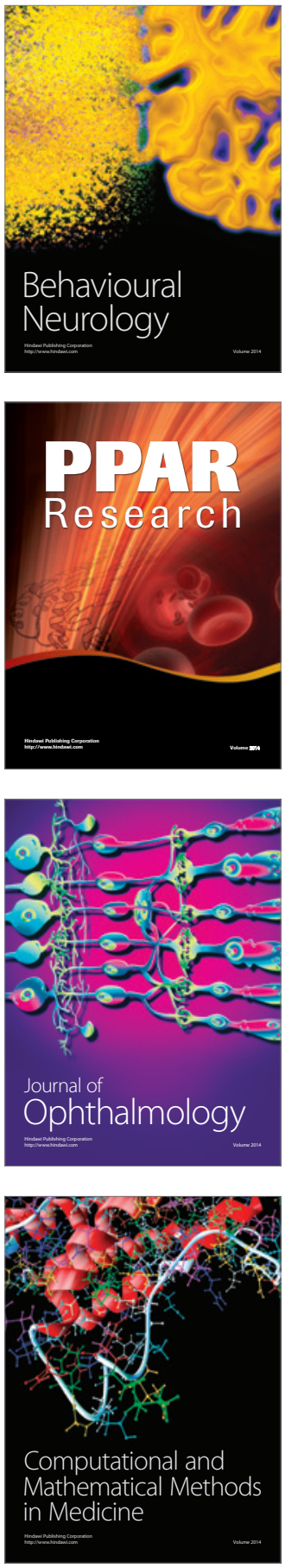

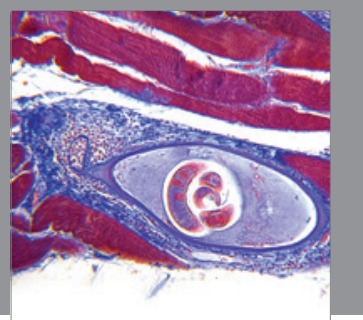

Gastroenterology

Research and Practice
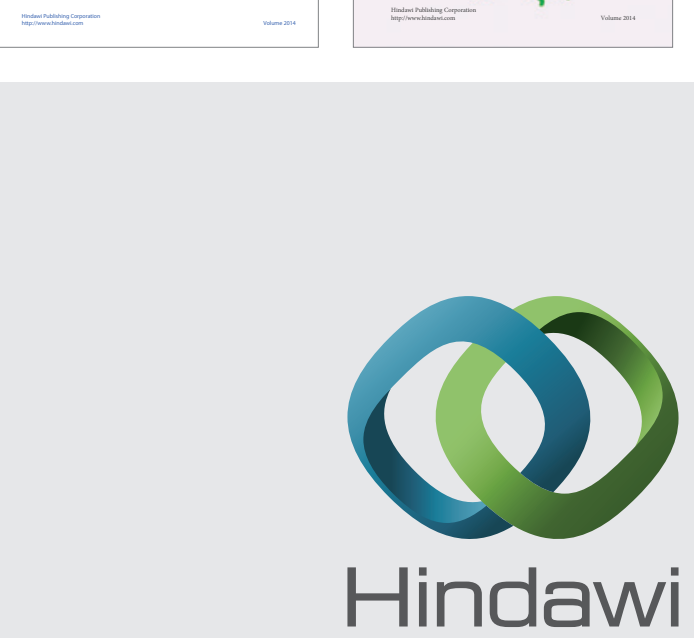

Submit your manuscripts at

http://www.hindawi.com
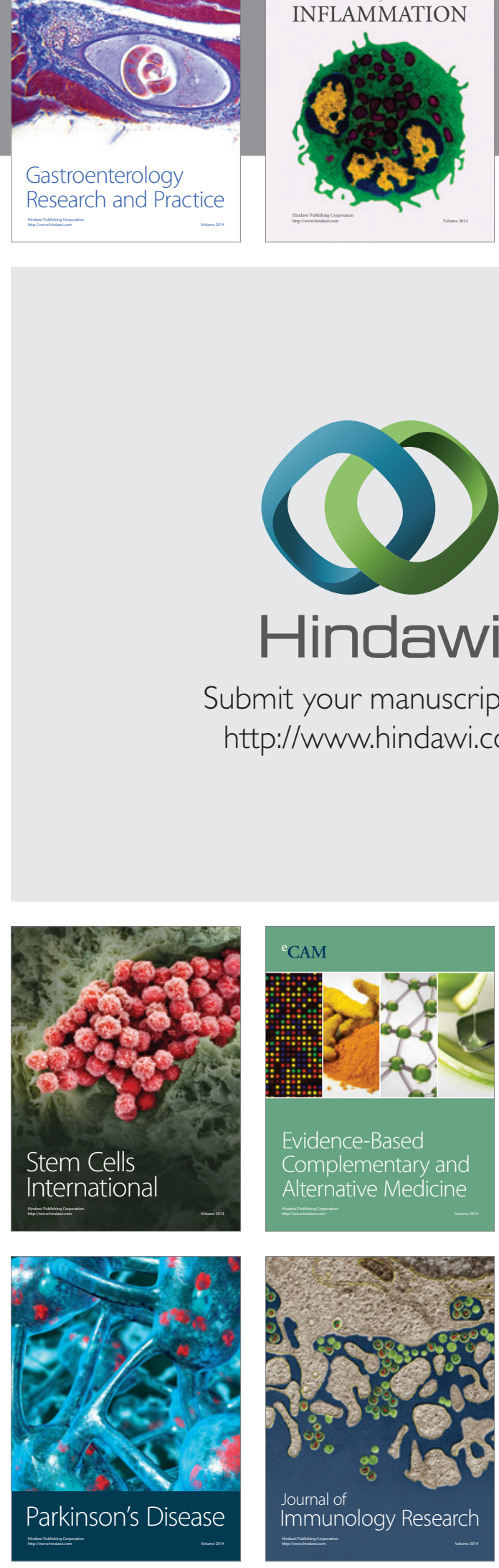

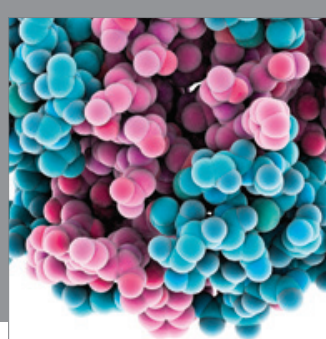

Diabetes Research
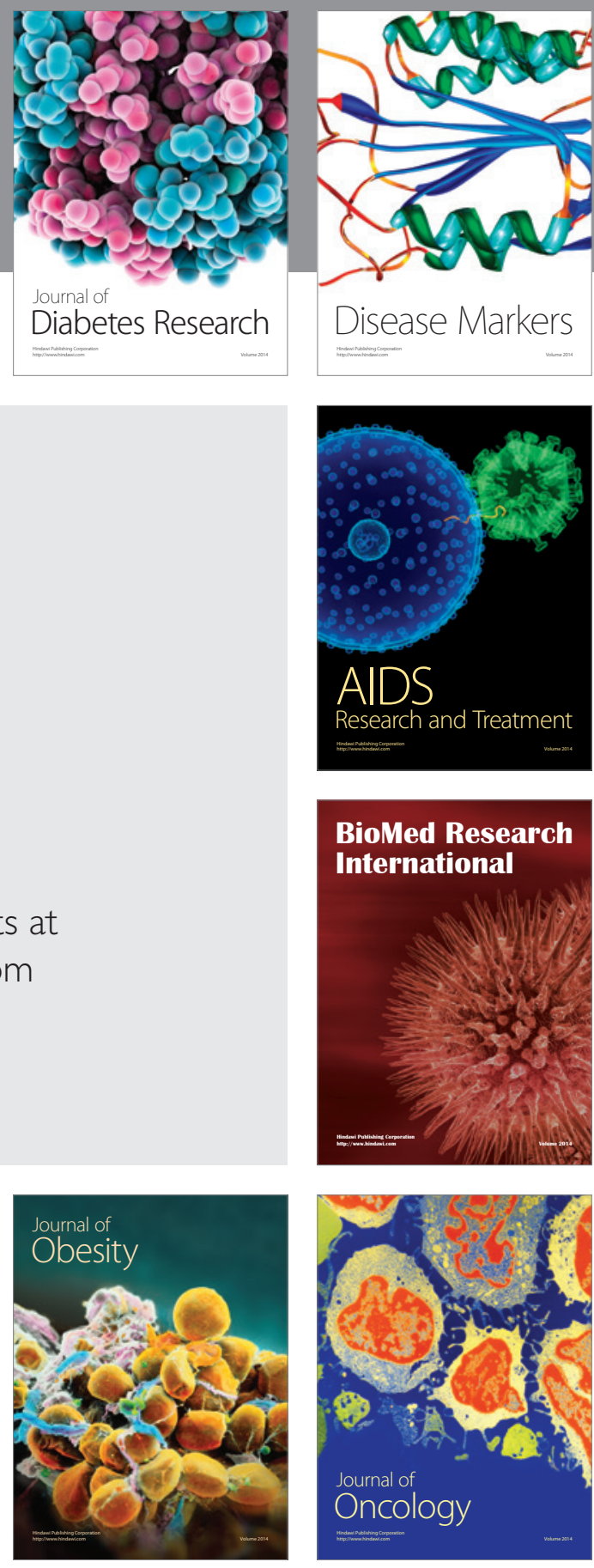

Disease Markers

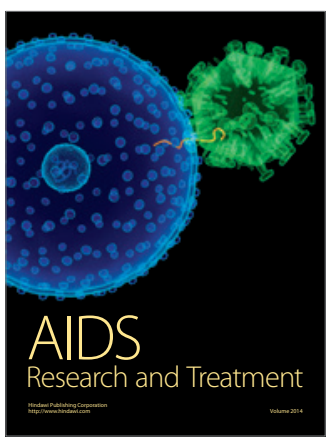

BioMed Research

International
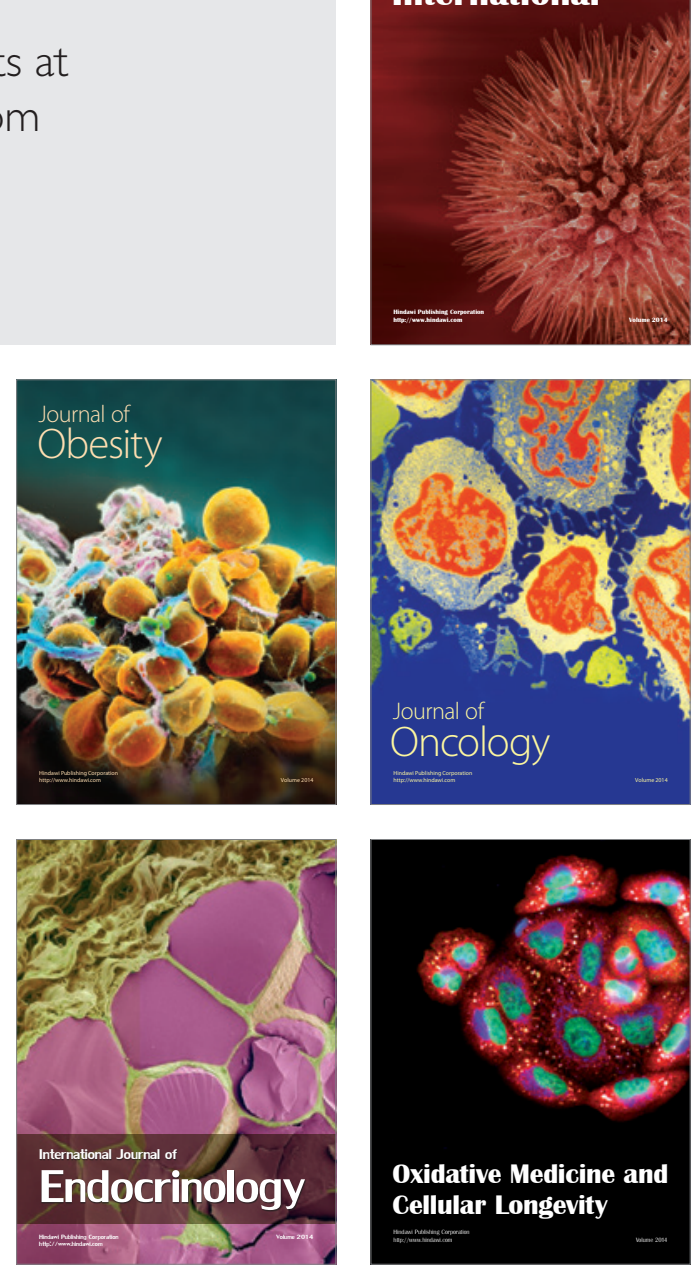